\title{
ENTRE CIENTIIFICOS DE DIFERENTES DISCIPLINAS SOCIALES, ¿SON SIMILARES LAS OPINIONES SOBRE LAS REGLAS DE INVESTIGACIÓN?
}

\author{
Alfredo de la Lama García, Marco de la Lama Zubirán, Marcelo del Castillo Mussot, Aline Magaña \\ Zepeda y Davide E. Daturi \\ UAM, UNAM, UAEM \\ México
}

\begin{abstract}
RESUMEN
El artículo responde a la interrogante “¿Es similar la distribución de las opiniones sobre las reglas de las investigación científica entre los investigadores de las diferentes disciplinas de ciencias sociales?”. Se realizó un estudio piloto de opiniones entre 137 investigadores de seis disciplinas de ciencias sociales de tres universidades públicas de México. Sus diferencias estadísticas frente al objetivo fueron analizadas mediante la prueba ji cuadrada. Los hallazgos prueban que entre los científicos sociales de diferentes disciplinas existen opiniones similares. Sin embargo, se presentan algunas discrepancias: "Estudiar la realidad" (entre psicólogos la aceptación es de $63 \%$, mientras que entre economistas es $93 \%$ ), y "Seguir el método", la aceptación de sociólogos e historiadores ( 61 y $50 \%$ respectivamente) contrasta con antropólogos (11\%) y administradores (27\%). Los científicos sociales divididos por disciplinas responden de manera similar tanto espontáneamente como si se les ayuda a recordar dichas reglas.
\end{abstract}

Palabras Clave:

ciencias sociales, educación superior, investigación científica, sociología de la ciencia, metodología.

\section{AMONG SCIENTISTS FROM DIFFERENT SOCIAL DISCIPLINES. ARE OPINIONS ABOUT THE RULES OF INVESTIGATION SIMILAR?}

\begin{abstract}
The article responds to the following question: "Is the distribution of opinions about the rules of scientific research similar among researchers in the different disciplines of social science?" A pilot study was conducted among 137 researchers from six social disciplines at three public universities in Mexico. Their statistical differences against the objective were analyzed using the Chi square test. The results show that among social scientists from different disciplines there are similar opinions on the rules of investigation. However, there are some discrepancies: "Studies reality as it is"; among psychologists the acceptance is $63 \%$, in contrast to economists (93\%). "Follow the scientific method": the acceptance of sociologists (61\%) and historians (50\%) contrasts with that of anthropologists (11\%) and business managers (27\%). Social scientists divided by disciplines respond in a similar way, eider spontaneously, as if they helps them remember those rules.
\end{abstract}

Keywords:

Health psychology, health promotion, disease prevention, models in health psychology, competencies in health psychology.

Bitácora del Artículo:

| Recibido: 1 de Febrero de 2018 | Aceptado: 1 Abril de 2018 | Publicado en línea: Enero-Junio de 2018 | 


\title{
ENTRE CIENTÍFICOS DE DIFERENTES DISCIPLINAS SOCIALES, ¿SON SIMILARES LAS OPINIONES SOBRE LAS REGLAS DE INVESTIGACIÓN?
}

\author{
Alfredo de la Lama García, Marco de la Lama Zubirán, Marcelo del Castillo Mussot, Aline Magaña \\ Zepeda y Davide E. Daturi \\ UAM, UNAM, UAEM \\ México
}

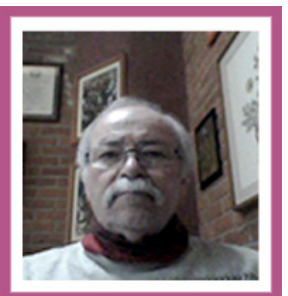

Alfredo de la Lama García

Depto. de Filosofía, UAM, Iztapalapa

Correo: adela2422@yahoo.com.mx

Economista y Dr. en Sociología por la UNAM, profesor investigador titular en la UAM-I. Miembro del Sistema Nacional de Investigadores, nivel I. Su interés académico gira en torno a la sociología de la ciencia y la historia económica global de los siglos XIX y XX.

Ver más...

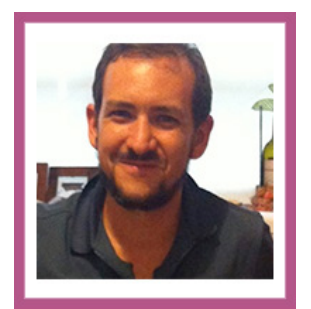

Marco de la Lama Zubirán Depto. de Economía, UAM, Iztapalapa Correo: marcodelazub@gmail.com

Profesor Asociado de la UAM, Iztapalapa, División de Ciencias Sociales y Humanidades (DCSH) del Departamento de Economía. Ingeniero, con maestría en mecánica en el Instituto de Ingeniería de la UNAM. Responsable del Sistema Divisional de Información y Planeación de la DCSH, Iztapalapa.

Ver más...

\section{CONTRIBUCIÓN DE LOS AUTORES}

Alfredo de la Lama diseñó y desarrolló el proyecto de investigación y su instrumento, coordinó los tres trabajos de campo y escribió los apartados de introducción, discusión y conclusiones | Marco de la Lama Zubirán se responsabilizó de escribir el método y hacer las operaciones de las pruebas de hipótesis. | Marcelo del Castillo Mussot. dirigió el trabajo de campo en la UNAM, codificó los resultados cuantitativos y los describió en el apartado de resultados. | Aline Magaña Zepeda. Dirigió el trabajo de campo en la UAM y codificó la pregunta abierta. | Davide E. Daturi. Dirigió el trabajo de campo en la UAEM. I Todos contribuyeron a la revisión del artículo en numerosas ocasiones, hasta que adquirió su forma final.

\section{AGRADECIMIENTOS}

Agradecemos el apoyo de la UNAM, a través del proyecto DGAPA IN101718

\section{Datos de FiLIACIÓN de los Autores}

Alfredo de la Lama García, Depto. de Filosofía, UAM, Iztapalapa | Marco de la Lama Zubirán, Depto. de Economía, UAM, Iztapalapa | Marcelo del Castillo Mussot. Instituto de Física, UNAM | Aline Magaña Zepeda, Depto. de Filosofía, UAM, Facultad de Economía, UNAM | Davide E. Daturi. Facultad de Filosofía. UAEM.

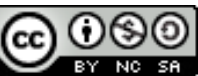

Copyright: @ 2018 de la Lama García, A., de la Lama Zubirán, M., del Castillo Mussot, M., Magaña Zepeda, A., \& Daturi, D. E.

Este es un artículo de acceso abierto distribuido bajo los términos de la licencia Creative Commons Reconocimiento-NoComercial -Compartirlgual 4.0 Internacional, por lo que su contenido gráfico y escrito se puede compartir, copiar y redistribuir total o parcialmente sin necesidad de permiso expreso de sus autores con la única condición de que no se puede usar con fines directamente comerciales y los términos legales de cualquier trabajo derivado deben ser los mismos que se expresan en la presente declaración. La única condición es que se cite la fuente con referencia a la Revista Digital Internacional de Psicología y Ciencia Social y a sus autores. 


\section{TABLA DE CONTENIDO}

INTRODUCCIÓN

MARCO TEÓRICO

Método

Participantes, 84

La muestra, 84

Materiales. El cuestionario, 84

Procedimiento. El trabajo de campo, 84

Mediciones, 85

Análisis estadísticos, 86

RESULTADOS

Las respuestas espontáneas de los investigadores sociales a la pregunta “¿Existen reglas en la investigación científica?”, 86

Revisión de los promedios de las respuestas a la pregunta 2, 87

Análisis de las respuestas por disciplina a la pregunta 2, 87

Análisis de las preguntas de recordación ayudada, 89

Discusión

CONCLUSIONES

Conclusiones por disciplinas, 92 


\section{INTRODUCCIÓN}

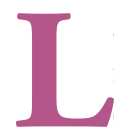
as universidades públicas tienen como una de sus principales finalidades practicar y difundir el conocimiento científico; incluso gente crtica que está convencida de este propósito denuncia que también son "Aparatos estatales para el poder", (Villoro Toranzó, 2014, pp. 5). Dicho conocimiento es aceptado por la comunidad científica gracias a un sistema específico que lo valida: la investigación científica. Habitualmente se cree que esta forma de conocer la realidad forma un todo coherente y en permanente desarrollo. Sin embargo, un reciente estudio empírico mostró que la investigación científica no es percibida de igual manera por todos los científicos; existen importantes diferencias entre los científicos naturales y sociales. Al parecer una quinta parte de los científicos sociales no coincide con dos de las cuatro reglas básicas que el resto dice seguir (De la Lama, Del Castillo y De la Lama, 2013).

Esta falta de homogeneidad en el pensamiento científico es un aliciente para indagar más a fondo estas discrepancias con el objetivo de tener un diagnóstico más certero del desarrollo de la educación superior, ahora que la "Sociedad del Conocimiento" (Thurow, 1996, pp. 92) se ha vuelto una realidad. Si se desea pertenecer a las culturas que están a la vanguardia de la civilización humana es necesario extender agresivamente el número de jóvenes que deseen practicar con éxito la investigación científica; y difundir sus reglas sobreentendidas, se estima, es un paso que lo facilitará.

El presente artículo buscar establecer si las opiniones de los científicos de las diferentes disciplinas sociales se distribuyen de forma similar respecto a las reglas de la investigación científica. Responder a esta interrogante permitirá conocer las similitudes y diferencias de la cultura científica de las instituciones públicas mexicanas de ciencias sociales. Un importante elemento que permite reflexionar respecto al papel que desempeñan los egresados de las ciencias sociales en el país. Por tal motivo, el objetivo de esta investigación busca responder a la siguiente pregunta: ¿Es similar la distribución de las opiniones sobre las reglas de la investigación científica entre los investigadores de las diferentes disciplinas de ciencias sociales?

\section{Marco teórico}

Filósofos, sociólogos, etnólogos y antropólogos, entre otros, han intentado identificar las reglas de la investigación científica. Por ejemplo, Merton (1973) considera que la comunidad científica tiene patrones que la hacen diferente al resto de las otras instituciones, lo que permite pensar que existe un Ethos (universalismo, comunismo, desinterés y escepticismo organizado) que protege a la ciencia de las fallas y limitaciones del resto de las otras organizaciones sociales. Las tesis de Merton han sido criticadas porque supuestamente corresponden a un programa normativo y no a las conductas efectivas de los científicos (Freeland, 2006; Pérez Tamayo, 2008). Se advierte que Merton (2016), en un artículo menos conocido planteó la teoría de las funciones manifiestas y latentes, donde destaca la existencia de una doble conducta para la educación. ¿Podría aplicarse también a la ciencia?

Bourdieu, sociólogo francés, se planta en una posición opuesta a la de Merton. Para el primero en la mayoría de los campos de la ciencia (laboratorios, institutos y disciplinas, entre otros), se necesitan recursos del exterior (privados y públicos) para realizar su trabajo, por ello se libra una lucha sin cuartel por el monopolio de la investigación científica entre los grandes capitales (simbólicos) —académicos muy reconocidos y autoridades-, "los que definen las reglas" (Bourdieu, 2000, pp. 80), y los pequeños productores - los jóvenes que entraron recién al campo-. Los primeros luchan porque no se transforme el campo; en cambio, los jóvenes tratan de hacer crecer su capital (simbólico) sin importar que en ocasiones se destruyan las reglas establecidas: "La diferencia entre un campo y un juego es que el primero es un juego en que las reglas mismas se ponen en juego, sea para consolidarlas sea para transformarlas" (Bourdieu, 2000, pp. 82).

Las reglas que propone Bourdieu, claramente son reglas de poder; por ende, son propias de cada institución o disciplina y, por tanto, no se refieren a las reglas generales que siguen los científicos cuando realizan su trabajo profesional. Debido a que Bourdieu privilegia las luchas de poder dentro de la ciencia acaba por impugnar el principio de que el mundo puede ser explicado por medio de la observación. Para refutar este principio, Bourdieu (2000, pp. 11) escribe: "[la] Verdad del producto [científico] reside en las particulares condiciones sociales de producción".

Del otro lado del canal, en otra escuela, la de Edimburgo, labora una serie de destacados antropólogos y etnólogos que han estudiado a la comunidad científica por medio de una teoría denominada constructivismo, la cual se halla entre las posiciones idealizadas de la ciencia de Merton y las confrontadas de Bourdieu. Para los 
constructivistas el conocimiento científico es resultado de una negociación entre los investigadores y los responsables de los proyectos — la autoridad-. El conocimiento científico se logra gracias a un consenso de la comunidad y no a exigentes pruebas capaces de ser replicadas. Latour (1987), por ejemplo, es enfático cuando afirma: "Por 'reglas de método' quiero decir qué decisiones a priori se deben tomar para considerar todos los hechos empíricos provistos por las disciplinas especializadas como parte del dominio de ciencia, tecnología y sociedad" (pp. 17). Knorr-Cetina (1999), otra destacada constructivista, aclara el sentido de dichas "decisiones", cuando sostiene una cultura epistémica: "Son las amalgamas de arreglos y mecanismos —alcanzados mediante la afinidad, necesidad y coincidencia histórica- que, en un campo dado, determina cómo conocemos y lo que conocemos" (pp. 1). Lo que significa, argumenta David (2006), que el reconocimiento de una teoría científica se logra en un contexto cultural específico, lo que ratifica que el contenido de la ciencia está construido socialmente.

Las aproximaciones de los constructivistas son interesantes, pero no se está de acuerdo con tres de sus elementos: 1) las reglas de la investigación científica no son decisiones a priori, son prácticas académicas que han sido transmitidas de una generación de científicos a otra, desde el siglo XVI (De la Lama, 2011), debido a que se han mostrado eficaces para resolver con éxito los complejos procesos internos de la investigación científica, que son descubrir, probar y comunicar abiertamente los resultados; 2) se omite que el investigador recurre a la actitud crítica para neutralizar cualquier efecto ajeno a las variables que estudia, aunque a veces no sea posible (Gould, 2004), y 3) desafortunadamente las reglas que han propuesto los constructivistas no tienen la aceptación de los científicos (Freeland, 2006; Pérez Tamayo 2008; Thuillier, 1991).

Este equipo de investigación buscó una aproximación diferente a nuestros predecesores. A partir de las evocaciones escritas de las experiencias en investigación de renombrados científicos se diseñaron cuatro reglas. La primera regla que se propone probar es "Estudiar la realidad tal cual" (Thuillier: 1991, pp. 8), esto porque la realidad tiene consistencias, regularidades y leyes que pueden ser observadas y comprendidas (Wigglesworth, 1987; Schrödinger, 1997). La segunda regla consiste en que el investigador desarrolle una actitud crítica frente a la investigación científica, concepto mencionado explícitamente por Popper (Holton, 1985) e indirectamente por otros científicos, como Medawar (en Holton, 1985); entendida dicha actitud como la convicción de que es indispensable hacerlo de manera objetiva, racional, sistemática y verificable. Actitud que permite tomar distancia de las preferencias, las obsesiones o los rechazos que se tengan respecto al objeto de estudio, sin importar los factores involucrados o los resultados encontrados. La tercera regla resume la facultad de pasar a la acción, la práctica; se trata de la capacidad para desarrollar procedimientos, técnicas e instrumentos capaces de efectuar las pruebas que permiten probar las hipótesis del estudio. "Naturalmente — dice Pérez Tamayo (2008, pp. 164) —, también había que dominar los aspectos técnicos del trabajo, el uso correcto de los aparatos de registro, la calibración basal para cada experimento, el diseño de controles adecuados, y otros cientos de detalles más que dependían directamente de nuestras habilidades y que también podían introducir 'ruido' en los resultados", y se sintetiza en un concepto nuevo: tener aptitud metodológica. La cuarta regla de la investigación científica demanda del científico nuevas habilidades, para que quede de manifiesto su disposición para comunicar de manera abierta los resultados de la investigación. Para ello desarrollará un lenguaje claro y preciso, de esta manera, eventualmente cualquier observador calificado podría someter a un examen crítico y verificar el estudio si se precisa (Hempel, 1988).

Las ciencias sociales representan una amplia variedad de disciplinas, y aunque todas ellas encuentran su valor científico en su acervo de datos, algunas tienen enfoques, teorías, métodos, procedimientos, técnicas e instrumentos diferentes al resto. Por ello, surge la interrogante de si las opiniones acerca de las reglas de la investigación científica se distribuyen de manera similar entre los científicos sociales de diferentes disciplinas. Para responder a esta problemática el presente estudio divide en seis categorías a los 137 científicos sociales encuestados de acuerdo con la disciplina que practican: Sociología [incluida Ciencia Política], Economía, Psicología junto con Psicología Social, Historia, Antropología y Administración [incluida Contaduría]).

La importancia de recoger opiniones para comprender el comportamiento de las sociedades, las comunidades y los individuos se sustenta en la ciencia de la conducta humana, la cual afirma que las opiniones, quizá, tienen relación estrecha con las actitudes e implican disposiciones para la acción y las conductas de las personas (Edward y Harold, 1980; Fiske, Gilbert y Lindzey, 2010).

Existe un problema adicional al desafío de recoger las opiniones de los investigadores respecto a las reglas investigadas advertido por científicos como Medawar (2013, pp. 32), que indica: "Los científicos observan sus reglas inconscientemente, y en el sentido de que no son capaces de expresarlo claramente en palabras, no lo saben", y Schrödinger (1997), quien aventuró una 
explicación para esta notable paradoja: la actitud fundamental hacia la ciencia "Se ha convertido en una actitud común, hasta el punto de olvidar que alguien tenía que planificar, hacer un programa y embarcarse en él" (pp. 80). Si ambos científicos tienen razón, los investigadores no mencionarán espontáneamente las reglas de la investigación científica, es decir, no existen reglas donde concuerde la mayoría de los científicos sociales. Este será el primer supuesto de esta investigación. Para resolver este contrasentido se recurrió a la teoría de los recuerdos auxiliados (aided recall). Esta teoría identifica y separa dos tipos de recuerdos cualitativamente diferentes: 1) los recuerdos espontáneos, los cuales son opiniones que libremente expresa el encuestado respecto al problema tratado por la investigación, y 2) los recuerdos ayudados, donde el encuestado es auxiliado para recordar el problema indagado (Danaher y Mullarkey, 2003; Reinares-Lara, Reinares-Lara \& Olarte-Pascual, 2016). Por tanto, el segundo supuesto de esta investigación plantea que las reglas de la investigación científica serán reconocidas por la mayoría de los investigadores de las ciencias sociales cuando sean mencionadas por el encuestador, lo que permite inferir que se trata de reglas sobreentendidas. Como tercer supuesto se afirma que existe un número estadísticamente significativo de científicos sociales que no reconocen estas cuatro reglas y que esta proporción diferirá en función de las diferentes disciplinas que componen el espectro de las ciencias sociales.

\section{MÉtodo}

\section{Participantes}

Para responder a las hipótesis de este trabajo era necesario pedir su opinión a personas que hicieran investigación en las diferentes disciplinas de ciencias sociales. La manera más expedita de encontrar a estas personas era buscarlas en las escuelas, facultades e institutos de investigación de ciencias sociales, y en este universo identificar a los individuos con más experiencia en investigación. Pareció que las personas más capacitadas para responder a nuestras inquietudes eran los investigadores o profesores-investigadores de tiempo completo que laboran en dichas instituciones. Una persona de estas características tiene dentro de sus obligaciones académicas realizar labores de investigación y enseñar a los alumnos este complejo trabajo. Nuestro equipo se centró en localizar instituciones de ciencias sociales y en ellas a los científicos que ahí laboran. Por tal motivo, la variable utilizada en esta investigación corresponde a los investigadores o profesores investigadores de tiempo completo de las instituciones de ciencias sociales.

\section{La muestra}

Una muestra representativa de una comunidad tan amplia suponía recorrer numerosas instituciones de educación superior, y eso estaba fuera de las posibilidades de los miembros de este grupo de investigadores. Es por ello que se decidió por una muestra piloto, no representativa e intencional (El Muestreo-Estadística, 2017; Weiers, 1986), que abarcara lo más ampliamente posible dicho universo en las limitaciones establecidas, es decir, encuestar sólo a investigadores y profesores-investigadores de tiempo completo de la escuela seleccionada; por tal motivo, los resultados son exploratorios y provisionales.

\section{Materiales. El cuestionario}

El cuestionario aplicado consta de una introducción donde se le explica al encuestado el objetivo y las personas que pretenden obtener su opinión, en los siguientes términos: "Somos un grupo interdisciplinario de estudiantes y especialistas de diversas universidades que estamos interesados en conocer las opiniones de los expertos en torno a su trabajo profesional. ¿Sería tan amable de contestar seis breves preguntas? Muchas gracias". A continuación se les hicieron seis preguntas para registrar dos tipos de recuerdos: los espontáneos y los auxiliados de acuerdo con la "teoría de la recordación ayudada" (Reinares-Lara, Reinares-Lara \& Olarte-Pascual, 2016: 257-278). La primera pregunta dividió a los científicos encuestados en dos grupos: los que no creen que existen reglas en la investigación científica, y los que opinan lo opuesto. Para los que respondieron que sí había reglas se hizo la pregunta 2: “¿Cuáles eran ellas?" Esta pregunta abierta permitió identificar las reglas que los científicos entrevistados expresaron libre y espontáneamente. Después a todos ellos, incluso a los que negaron la existencia de reglas, se les formularon otras cuatro preguntas dirigidas a que opinaran y WWran las cuatro reglas que el estudio proponía. El cuestionario se encuentra en el apéndice.

\section{Procedimiento. El trabajo de campo}

Para probar los supuestos de este estudio se solicitó a 141 científicos de tiempo completo que respondieran un cuestionario (véase el apéndice); cuatro (2.9\%) se negaron a responderlo, por lo que el total de encuestados fue de 137 (97.2\%). El trabajo de campo se hizo en tres universidades públicas, dos en la Ciudad de México (Universidad Autónoma Metropolitana, Iztapalapa [UAM-I], División de Ciencias Sociales y Humanidades, 
y la Universidad Nacional Autónoma de México, Ciudad Universitaria [UNAM], en sus facultades de Economía, Ciencias Políticas y Sociales, Psicología, y los institutos de Economía y Antropología), y la Universidad Autónoma del Estado de México (UAEM), en sus facultades de Ciencias Políticas y Sociales, Economía, Historia, Administración y Psicología, entre los meses de febrero y mayo de 2012 y mayo a septiembre de 2013. El ambiente en que se efectuó el trabajo de campo fue receptivo; incluso numerosos encuestados incorporaron observaciones no solicitadas para reafirmar sus respuestas y contribuyeron a enriquecer este informe. Las escuelas, facultades e institutos donde se realizó el trabajo de campo fueron 17. La distribución por escuela y disciplinas de ciencias sociales de los científicos encuestados se muestra en la tabla 1. Estudiantes universitarios entrenados y pagados aplicaron los cuestionarios a personas que afirmaron ser investigadores de tiempo completo, localizados en cubículos, salones y laboratorios de las instituciones referidas.

Tabla 1.

Encuestados por universidad y facultades o institutos de Ciencias Sociales

\begin{tabular}{|c|c|c|c|c|}
\hline Disciplinas & UAM & UNAM & UAEM & TOTAL \\
\hline $\begin{array}{l}\text { Sociología y Ciencias } \\
\text { Políticas }\end{array}$ & 10 & 10 & 8 & 28 \\
\hline $\begin{array}{l}\text { Administración y } \\
\text { Contaduría }\end{array}$ & 10 & 7 & 5 & 22 \\
\hline $\begin{array}{l}\text { Psicología y } \\
\text { Psicología Social }\end{array}$ & 10 & 10 & 10 & 30 \\
\hline Economía & 10 & 10 & 8 & 28 \\
\hline Antropología & 6 & 3 & 0 & 9 \\
\hline Historia & 9 & 0 & 11 & 19 \\
\hline $\begin{array}{l}\text { Total de } \\
\text { encuestados }\end{array}$ & 55 & 40 & 42 & 137 \\
\hline
\end{tabular}

Respecto al nivel de estudios que dicen tener los encuestados predominan los doctorados, y en segundo lugar las maestrías; su distribución es semejante en las tres universidades, aunque la UAEM destaca ligeramente (tabla 2).
Tabla 2.

Nivel de estudios de los científicos encuestados

\begin{tabular}{lc|c|c|c|}
\multicolumn{1}{r}{ Estudios } & UAM & \multicolumn{1}{c}{ UNAM } & \multicolumn{1}{c}{ UAEM } & TOTAL \\
\hline Doctorado & $75 \%$ & $75 \%$ & $81 \%$ & $76 \%$ \\
\hline Maestría & $20 \%$ & $20 \%$ & $19 \%$ & $22 \%$ \\
\hline Licenciatura & $5 \%$ & $5 \%$ & $0 \%$ & $2 \%$ \\
\hline Total & $100 \%$ & $100 \%$ & $100 \%$ & $100 \%$ \\
\hline Encuestados & 55 & 40 & 42 & 137 \\
\hline
\end{tabular}

Fuente: Sondeo de opinión entre 137 científicos de la UNAM, la UAEM y la UAM.

En cuanto al origen de sus últimos estudios, $75 \%$ de los encuestados dicen que los hicieron en alguna universidad de México. La UAEM es la institución que tiene la mayor diversidad y la UNAM la más excluyente. El resto de los encuestados afirma haber efectuado sus últimos estudios en instituciones extranjeras; las facultades europeas predominan sobre las estadounidenses (tabla 3 ).

Tabla 3.

Lugar de los últimos estudios de los científicos encuestados

\begin{tabular}{|c|c|c|c|}
\hline UBICACIÓN & UAM & UNAM & UAEM \\
\hline UNAM & $25 \%$ & $71 \%$ & $10 \%$ \\
\hline UAM & $19 \%$ & $0 \%$ & $7 \%$ \\
\hline UAEM & $0 \%$ & $0 \%$ & $31 \%$ \\
\hline $\begin{array}{l}\text { OtRAS } \\
\text { INSTITUCIONES } \\
\text { NACIONALES }\end{array}$ & $31 \%$ & $6 \%$ & $26 \%$ \\
\hline UNIÓN EUROPEA & $15 \%$ & $16 \%$ & $24 \%$ \\
\hline USA Y CANADÁ & $10 \%$ & $6 \%$ & $0 \%$ \\
\hline TOTAL & $100 \%$ & $100 \%$ & $100 \%$ \\
\hline ENCUESTADOS & 55 & 40 & 42 \\
\hline
\end{tabular}

\section{Mediciones}

Para determinar las diferencias en la aceptación de las reglas de la investigación científica los investigadores encuestados se dividieron por disciplinas: Economía, Sociología (incluye a Ciencias Políticas), Psicología (incluida Psicología Social), Antropología, Historia y Administración (que engloba Contaduría) y así determinar si existían diferencias entre ellos. 


\section{Análisis estadísticos}

Las respuestas de los encuestados por disciplinas se sometieron a un análisis estadístico con la finalidad de establecer si las diferencias encontradas son producto del azar o si es posible aceptar otra explicación.

Para el análisis estadístico de las respuestas se consideró que las respuestas a cada pregunta son dicotómicas y el número de casos 137 . Esto permite hacer una comparación de los resultados por medio de la prueba ji cuadrada, en concordancia con las sugerencias para las pruebas de hipótesis de Siegel y Castellan (1998): "Cuando $\mathrm{N}>40$, utilice la prueba $X^{2}$, corregida para la continuidad"; posee la característica de abordar problemas que se expresan en una tabla $2 \times 2$, donde hay dos variables dicotómicas y una escala nominal (todas las tablas $2 \times 2$ tienen sólo un grado de libertad $[G L]=1$ ).

$$
X^{2}=\frac{N\left(|A D-B C|-\frac{N}{2}\right)^{2}}{(A+B)(C+D)(A+C)(B+D)}
$$

Tabla 4.

Tabla de contingencia de la prueba $X^{2}$ corregida para la continuidad

\begin{tabular}{|c|c|c|c|}
\hline \multicolumn{5}{|c|}{ TABLA DE CONTINGENCIA $2 \times 2$} \\
\hline Variable & Grupo 1 & Grupo 2 & Combinación \\
\hline Sí & A & B & A + B \\
\hline No & C & D & C + D \\
\hline Total & A + C & B + D & N \\
\hline
\end{tabular}

La X2 obtenida por la fórmula se compara con el límite o frontera establecida por la prueba ji cuadrada. Si X2 tiene un valor $\leq$ a 3.84 se acepta que las variaciones encontradas son producto del azar, pero si es mayor a este número se rechaza la hipótesis nula y se acepta que el proceso estudiado tiene otra explicación. En esta prueba se reconoce que puede haber un error de $5 \%$ en los resultados y que las casillas no deben contener una frecuencia menor a 5 (Siegel y Castellan, 1998). A partir de la tabla 6 se encuentran las pruebas que se mencionan en los resultados.

\section{Resultados}

El análisis de las respuestas se divide en dos categorías: las afirmaciones dadas por los investigadores espontánea y libremente, y las respuestas que dieron los científicos encuestados cuando se les preguntó si las reglas propuestas por esta investigación eran reglas de la investigación científica.

\section{Las respuestas espontáneas de los investigadores sociales a la pregunta “¿Existen reglas en la investigación científica?"}

Las respuestas espontáneas a la pregunta 1 dividieron a los científicos sociales en dos grupos: los que no creen que haya reglas, un poco más de uno de cada cinco encuestados y el resto. Los encuestados divididos por disciplinas sociales se dividen en tres grupos: 1) los que en una proporción mayor rechazan que haya reglas (economistas, administradores y contadores); 2) los que tienen una posición contraria y aceptan en su mayoría que en efecto hay reglas (sociólogos, incluidos politólogos y antropólogos), y 3) los que se encuentran en una posición intermedia (Psicología e Historia) (figura 1).

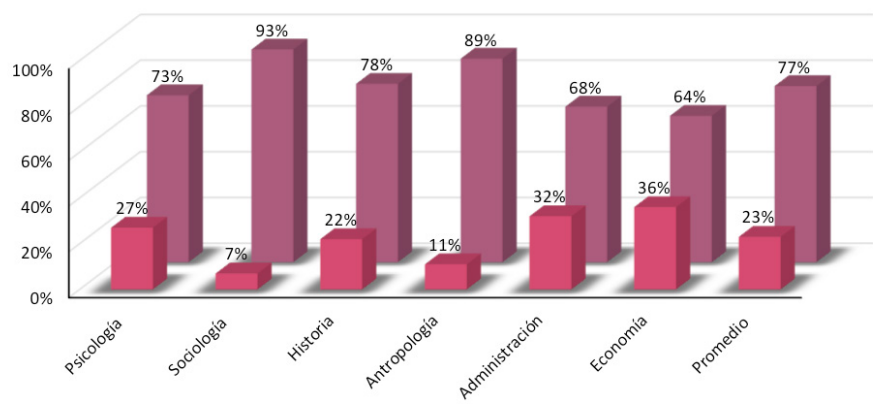

No hay reglas $\quad$ Hay reglas

Figura 1.

Respuestas a la pregunta 1. En su opinión, ¿cree que existan reglas o acuerdos dentro de la investigación científica que sigan la mayoría de los científicos en activo?

A las respuestas de los investigadores encuestados de Sociología y Ciencias Políticas se aplicó la prueba de ji cuadrada; el resultado es el siguiente: $\mathrm{X} 2=6.369$; como es mayor al valor crítico de ji cuadrada $=3.84$, con un $G L=1$, y un error de 5\%, se rechaza la hipótesis nula; las diferencias tienen otra explicación (tabla 9).

También se aplicó el test a los economistas encuestados X2 $=2.196$. Dado que el límite de ji cuadrada es 3.84, cuando hay un GL $=1$ con un error de $95 \%$, no existen elementos para rechazar la hipótesis nula; sin embargo, se hace notar la cercanía al límite, por lo que estas conclusiones deben tomarse con reserva (tabla 18).

A los antropólogos e historiadores encuestados no se les pudo aplicar el test de la X2 porque una de sus casillas tiene menos frecuencias de las que demanda la prueba. El resto de los encuestados, divididos por disciplinas, mantienen una división de sus respuestas semejante al promedio, por lo que es posible suponer que sus variaciones se deben al azar. 
La pregunta 2 ("¿Podría mencionar algunas de estas reglas o acuerdos?") se aplicó sólo a los científicos encuestados que opinaron que sí había reglas.

La codificación de las respuestas abiertas fue independiente para cada regla e incluyó a los que dijeron encuestados. El resto de las reglas apenas son mencionadas (entre 7 y $2 \%$ ). Las menciones de reglas que prácticamente no se repitieron alcanzó poco más de uno de cada cinco investigadores encuestados (tabla 5).

Tabla 5.

Respuestas a la pregunta 2. ¿Podría mencionar algunas de estas reglas o acuerdos?

\begin{tabular}{|c|c|c|c|c|c|c|c|}
\hline $\begin{array}{c}\text { Reglas } \\
\text { MENCIONADAS }\end{array}$ & Psicología & Sociología* & Historia & ANTROPOLOGÍA & ADMINISTRACIÓN $* *$ & ECONOMÍA & Promedio \\
\hline $\begin{array}{l}\text { Seguir el método } \\
\text { científico }\end{array}$ & $40 \%$ & $61 \%$ & $50 \%$ & $11 \%$ & $27 \%$ & $39 \%$ & $41 \%$ \\
\hline $\begin{array}{l}\text { Tener actitud } \\
\text { crítica }\end{array}$ & $20 \%$ & $21 \%$ & $33 \%$ & $0 \%$ & $9 \%$ & $14 \%$ & $18 \%$ \\
\hline $\begin{array}{l}\text { Seguir } \\
\text { reglamentos } \\
\text { institucionales }\end{array}$ & $20 \%$ & $18 \%$ & $11 \%$ & $33 \%$ & $18 \%$ & $11 \%$ & $17 \%$ \\
\hline $\begin{array}{l}\text { Publicar los } \\
\text { resultados }\end{array}$ & $17 \%$ & $7 \%$ & $6 \%$ & $22 \%$ & $9 \%$ & $11 \%$ & $11 \%$ \\
\hline $\begin{array}{l}\text { Tener aptitud } \\
\text { metodológica }\end{array}$ & $7 \%$ & $18 \%$ & $0 \%$ & $0 \%$ & $9 \%$ & $4 \%$ & $7 \%$ \\
\hline $\begin{array}{l}\text { Formar recursos } \\
\text { humanos }\end{array}$ & $0 \%$ & $4 \%$ & $6 \%$ & $0 \%$ & $9 \%$ & $11 \%$ & $5 \%$ \\
\hline $\begin{array}{l}\text { Tener ética o } \\
\text { bioética }\end{array}$ & $23 \%$ & $0 \%$ & $0 \%$ & $11 \%$ & $0 \%$ & $4 \%$ & $7 \%$ \\
\hline $\begin{array}{l}\text { Hacer } \\
\text { contribuciones } \\
\text { originales }\end{array}$ & $0 \%$ & $4 \%$ & $11 \%$ & $0 \%$ & $0 \%$ & $4 \%$ & $3 \%$ \\
\hline $\begin{array}{l}\text { Estudiar la } \\
\text { realidad }\end{array}$ & $3 \%$ & $4 \%$ & $0 \%$ & $0 \%$ & $0 \%$ & $4 \%$ & $2 \%$ \\
\hline Otras reglas & $23 \%$ & $21 \%$ & $33 \%$ & $33 \%$ & $9 \%$ & $21 \%$ & 22 \\
\hline \multicolumn{8}{|c|}{ * Incluye a encuestados de Ciencias Políticas. } \\
\hline \multicolumn{8}{|c|}{ ** Incluye a encuestados de Contaduría. } \\
\hline Fuente: Sondeo de & ión entre & científicos & la UNAI & UAEM y la & & & \\
\hline
\end{tabular}

que no había reglas. Esto es, se sumaron los que mencionaron la regla en cuestión más los científicos encuestados que no la mencionaron, más los que dijeron que no había reglas. El resultado permite obtener la distribución de los pesos relativos para cada regla de manera independiente, dentro del conjunto de los investigadores encuestados (137).

\section{Revisión de los promedios de las respuestas a la pregunta 2}

En términos generales la regla "Seguir el método científico" fue la respuesta espontánea que registró más frecuencia (41\%). Le sigue en segundo nivel "Tener actitud crítica" y "Seguir los reglamentos institucionales", pero la frecuencia de menciones es muy inferior (18 y 17\%). En cuarto lugar se encuentra la regla "Publicar los resultados"; sólo la mencionan uno de cada 10 investigadores
Análisis de las respuestas por disciplina a la pregunta 2

Los psicólogos encuestados tienen una opinión parecida a los promedios encontrados por sus colegas de otras disciplinas respecto a las reglas manifestadas de manera espontánea, excepto en una de ellas. Casi uno de cada cuatro psicólogos dicen que "Tener ética o bioética" es una regla de su práctica científica, en tanto que el resto de los investigadores encuestados de las otras disciplinas no la mencionan.

Aplicada a la disciplina de Psicología, la prueba ji cuadrada para la regla "Tener bioética o ética" el resultado es X2 $=14.26$, superior a X2 $=3.86$, con GL $=1$ y un error de $5 \%$; en consecuencia, se rechaza la hipótesis nula; las diferencias no están dadas por el azar (tabla 6).

Para los investigadores de Sociología y Ciencias Políticas encuestados existen tres reglas de la investigación científica que fueron mencionadas espontáneamente y que difieren del promedio general; dos están por arriba 
de él ("Seguir el método científico" y "Tener aptitud metodológica") y una por debajo ("Tener que publicar"). Se aplicó la prueba de X2 a las dos primeras reglas; los resultados son: $\mathrm{X} 2=6.57$ y 6.37 . A la tercera regla no se le aplicó la prueba porque una de sus casillas tuvo una frecuencia menor a 5. Los resultados de las dos primeras reglas son mayores al valor crítico de ji cuadrada $=3.84$, con un $G L=1$, y un error de 5\%; por tanto, se rechazan las hipótesis nulas en estos casos (tablas 9-10 y 11).

Los científicos encuestados de Historia tienen una distribución similar respecto a los promedios encontrados en la mayoría de las reglas manifestadas espontáneamente; sin embargo, hay dos reglas que salen del promedio ("Tener actitud crítica" y "Hacer contribuciones originales"); una diferencia de 15 y $7 \%$ respectivamente (tabla 5).

La prueba de ji cuadrada para la regla "Tener actitud crítica" aplicada a los historiadores es X2 = 0.999; como el valor límite de ji cuadrada $=3.84$, con un GL $=1$ y $5 \%$ de error, se concluye que las diferencias son aleatorias. Para la regla "Hacer contribuciones originales" no se hizo la prueba porque no se cumplió una de las condiciones: que las casillas tengan más de cinco frecuencias (tablas 12-13).

Los científicos encuestados de Antropología presentan los valores extremos en cuatro reglas mencionadas espontáneamente; "Seguir el método científico" y "Tener actitud crítica" tienen valores menores al resto de sus colegas de Ciencias Sociales; por otra parte, "Seguir leyes y reglamentos institucionales" y "Tener que publicar" tienen las proporciones más altas (tabla 5).

Las pruebas ji cuadrada para las cuatro reglas mencionadas no se hicieron porque no se cumple con la condición de que una de las casillas de la prueba tenga más de cinco frecuencias (tablas 14-17).

Los investigadores de Administración y Contaduría encuestados tal vez son los más representativos de los promedios de todas las reglas de la investigación científica que se mencionan espontáneamente. Por ello, no es necesario realizar la prueba ji cuadrada porque no hay variaciones importantes respecto al resto de los otros encuestados.

Sin embargo, destaca una paradoja: $27 \%$ de los encuestados de esta disciplina opinan espontáneamente que una regla es "Seguir el método científico", mientras que otra proporción, un poco mayor (32\%), afirma en la pregunta inicial que "No hay reglas" en la investigación científica (tabla 5, figura 1).

Los científicos de Economía encuestados se parecen en sus respuestas espontáneas a los de Administración y Contaduría, es decir, son similares al promedio general. En consecuencia, se consideró que las diferencias en- contradas entre los economistas encuestados frente al resto de la muestra son producto del azar.

Entre estos profesionales encuestados también se presenta la contradicción de que una alta proporción se manifiesta a favor de la regla "Seguir el método científico" (39\%), en tanto que otra proporción semejante $(36 \%)$ inicialmente niega que haya reglas en la investigación científica (tabla 5 , figura 1 ).

Tabla 6.

$X^{2}$ para el acuerdo "Tener ética o bioética"

\begin{tabular}{|c|c|c|c|}
\hline Lo MENCIONAN & C. SOCIALES & SOCIOLOGíA & COMBINACIÓN \\
\hline NO & 105 & 23 & 128 \\
\hline Sí & 2 & 7 & 9 \\
\hline Total & 107 & 30 & 137 \\
\hline
\end{tabular}

Tabla 7.

$X^{2}$ para la pregunta 3 ("Estudiar la realidad")

\begin{tabular}{|c|c|c|c|}
\hline Lo MENCIONAN & C. SOCIALES & SOCIOLOGíA & COMBINACIÓN \\
\hline Sí & 89 & 19 & 108 \\
\hline No & 18 & 11 & 29 \\
\hline Total & 107 & 30 & 137 \\
\hline
\end{tabular}

Tabla 8.

$X^{2}$ para la regla "Seguir el método científico"

\begin{tabular}{|c|c|c|c|}
\hline LO MENCIONAN & C. SOCIALES & SOCIOLOGíA & COMBINACIÓN \\
\hline NO & 58 & 23 & 81 \\
\hline Sí & 51 & 5 & 56 \\
\hline Total & 109 & 28 & 137 \\
\hline
\end{tabular}

Tabla 9.

$X^{2}$ para "No hay reglas en la investigación"

\begin{tabular}{|c|c|c|c|}
\hline LO MENCIONAN & C. SOCIALES & SOCIOLOGíA & COMBINACIÓN \\
\hline NO & 85 & 20 & 105 \\
\hline Sí & 24 & 8 & 32 \\
\hline Total & 109 & 28 & 137 \\
\hline
\end{tabular}

Tabla 10.

$X^{2}$ para "Tener aptitud metodológica"

\begin{tabular}{|c|c|c|c|}
\hline LO MENCIONAN & C. SOCIALES & SOCIOLOGía & COMBINACIÓN \\
\hline NO & 104 & 23 & 127 \\
\hline Sí & 5 & 5 & 10 \\
\hline Total & 109 & 28 & 137 \\
\hline
\end{tabular}


Tabla 11.

$X^{2}$ para "Tener que publicar"

\begin{tabular}{|c|c|c|c|}
\hline Lo MENCIONAN & C. SOCIALES & Sociología & Combinación \\
\hline NO & 96 & 26 & 122 \\
\hline Sí & 13 & 2 & 15 \\
\hline Total & 109 & 28 & 137 \\
\hline
\end{tabular}

Tabla 12.

$X^{2}$ para la pregunta 1 ("¿Hay reglas?"

\begin{tabular}{|c|c|c|c|}
\hline Lo mencionan & \multicolumn{1}{c}{ C. SOCIALES } & SOCIOLOGía & Combinación \\
\hline Lo mencionan & C. Sociales & Historia & Combinación \\
\hline Sí las hay & 89 & 16 & 135 \\
\hline No las hay & 28 & 4 & 32 \\
\hline Total & 119 & 18 & 137 \\
\hline
\end{tabular}

Tabla 13.

$X^{2}$ para "Tener actitud crítica"

\begin{tabular}{|c|c|c|c|}
\hline Lo mencionan & C. SOCIALES & SOCIOLOGía & COMBINACión \\
\hline Lo mencionan & C. Sociales & Historia & Combinación \\
\hline No & 101 & 12 & 113 \\
\hline Sí & 18 & 6 & 24 \\
\hline Total & 119 & 18 & 137 \\
\hline
\end{tabular}

Tabla 14.

$X^{2}$ para "Seguir el método científico"

\begin{tabular}{|c|c|c|c|}
\hline Lo mencionan & C. SOCIALES & Sociología & Combinación \\
\hline No & 73 & 8 & 81 \\
\hline Sí & 55 & 1 & 56 \\
\hline Total & 128 & 9 & 137 \\
\hline
\end{tabular}

Tabla 15.

$X^{2}$ para el acuerdo "Tener ética o bioética"

\begin{tabular}{|c|c|c|c|}
\hline Lo MENCIONAN & C. SOCIALES & SOCIOLOGía & COMBinaCión \\
\hline No & 104 & 9 & 113 \\
\hline Sí & 24 & 0 & 24 \\
\hline Total & 128 & 9 & 137 \\
\hline
\end{tabular}

Tabla 16.

$X^{2}$ para "Seguir reglamentos institucionales"

\begin{tabular}{|c|c|c|c|}
\hline Lo MENCIONAN & C. SOCIALES & SOCIOLOGía & COMBINACión \\
\hline No & 108 & 6 & 114 \\
\hline Sí & 20 & 3 & 23 \\
\hline Total & 128 & 9 & 137 \\
\hline
\end{tabular}

Tabla 17.

$X^{2}$ para "Tener que publicar"

\begin{tabular}{|c|c|c|c|}
\hline Lo MENCIONAN & C. SOCIALES & SOCIOLOGía & COMBINACIÓN \\
\hline No & 113 & 7 & 120 \\
\hline Sí & 13 & 2 & 15 \\
\hline Total & 126 & 9 & 135 \\
\hline
\end{tabular}

Tabla 18.

$X^{2}$ para "No hay reglas en la investigación"

\begin{tabular}{|c|c|c|c|}
\hline Lo mencionan & C. Sociales & Sociología & Combinación \\
\hline No & 87 & 18 & 105 \\
\hline Sí & 22 & 10 & 32 \\
\hline Total & 109 & 28 & 137 \\
\hline
\end{tabular}

Tabla 19.

$X^{2}$ para la pregunta 3 ("Estudiar la realidad")

\begin{tabular}{|c|c|c|c|}
\hline Lo mencionan & C. Sociales & Sociología & Combinación \\
\hline Sí & 82 & 26 & 108 \\
\hline No & 27 & 2 & 29 \\
\hline Total & 109 & 28 & 137 \\
\hline
\end{tabular}

\section{Análisis de las preguntas de recordación ayudada}

Las preguntas 3 a 6 del cuestionario se hicieron al total de los científicos, incluidos los que originalmente afirmaron que no existen reglas en la investigación científica, por lo que el número total de encuestados no se modifica (137). La razón para proceder de esta manera obedece a que si los encuestados están convencidos de que no existen reglas en la investigación científica, mantendrán las respuestas negativas cuando se les planteen dichas preguntas.

La pregunta 3 propone: " $i$ Cree que una de las reglas sea pensar que en la naturaleza (o en la sociedad, en su caso) existen leyes o regularidades que pueden ser explicadas a través de la observación y el razonamiento?" La mayoría de las respuestas de los científicos sociales en- 
cuestados, por cada disciplina analizada, son afirmativas; su promedio es $79 \%$, excepto para los psicólogos y los economistas (63 y 93 respectivamente) (figura 2).

Aplicada a los psicólogos encuestados, la prueba ji cuadrada para la regla "Estudiar la realidad" obtuvo X2 $=4.40$, superior a $\mathrm{X} 2=3.86$, con un $\mathrm{GL}=1$ y un error de 5\%; en consecuencia, se rechaza la hipótesis nula; las diferencias no están dadas por el azar (tabla 7).

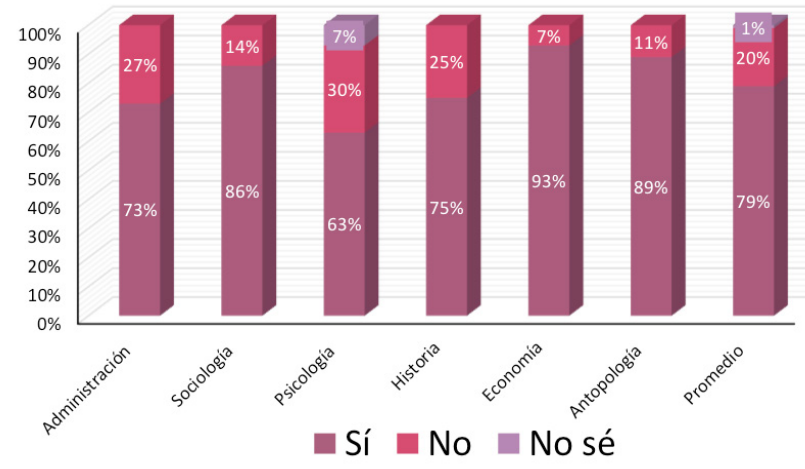

Figura 2.

Respuesta a la pregunta 3. ¿Cree que una de las reglas sea pensar que en la naturaleza (o en la sociedad, en su caso) existen leyes o regularidades que pueden ser explicadas a través de la observación y el razonamiento?

La prueba de hipótesis de la variable "Estudiar la realidad" hecha a los economistas encuestados arroja una $\mathrm{X} 2=3.16$, con un $\mathrm{GL}=1$, y un error de $5 \%$; entonces no existen elementos para rechazar la hipótesis nula; sin embargo, se hace notar la cercanía al límite, por lo que estas conclusiones deben tomarse con reserva (tabla 19).

Las opiniones expresadas que apoyan la afirmación de que la ciencia estudia la realidad tal como es, son: "Es una regla universal: La realidad existe independientemente del observador", "Se confronta la investigación con la realidad", o "Se contrasta la teoría con la realidad".

Las opiniones en contra de esta regla son: "No es estrictamente una regla", "Hay un grupo para quien sí cumple y para quien no", "Sí hay acuerdos, pero reglas aplicadas al ámbito social no son una constante", o bien "Las leyes no existen, lo cual no quiere decir que la observación y el razonamiento no sean útiles".

La pregunta 4 del cuestionario es: " $i$ Estima que otra de las reglas sería que el investigador tenga una actitud crítica frente al objeto de estudio?" Es decir, que desarrolle la capacidad de analizar de manera objetiva, racional, verificable y sistemática la información contenida en toda investigación.

Las respuestas afirmativas a la pregunta 4 de los investigadores encuestados tienen en promedio $81 \%$; por el contrario, 19\% de ellos negaron que la actitud crítica, como la define la pregunta 4, sea una regla que norme la actividad investigativa en las ciencias sociales. El rechazo más significativo a esta regla lo manifiestan los sociólogos junto con los politólogos (29\%), y los que más la aprecian son los antropólogos (89\% de aceptación). El resto de las disciplinas tiene promedios muy semejantes (81\%) (figura 3).

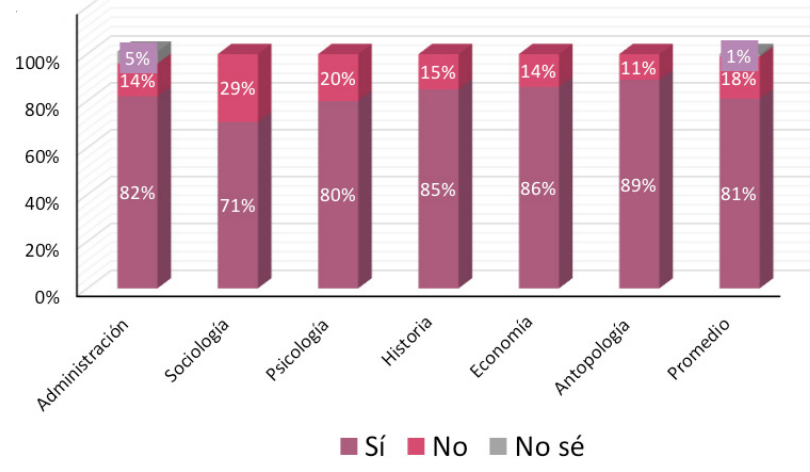

Figura 3.

Respuesta a la pregunta 4. ¿Estima que otra de las reglas sería que el investigador tenga actitud crítica frente al objeto de estudio? Es decir, ¿que desarrolle la capacidad de analizar de manera objetiva, racional, verificable y sistemática la información contenida en toda investigación?

El análisis de la ji cuadrada a las respuestas de los científicos de la disciplina de Ciencias Políticas y Sociales es X2 $=7.93$, como el límite de la ji cuadrada $=3.84$, con un $\mathrm{GL}=$ 1, con 95\% de confiabilidad, se rechaza la hipótesis nula y se acepta que su rechazo va más de la casualidad.

Los encuestados que favorecen esta regla recuerdan que en esta regla se encierra uno de los valores básicos de la ciencia: "Sí, es un 'mito fundamental'", "Es indispensable", "Diferencia entre un investigador y un cientificista o aficionado a la ciencia"; otros testimonios mencionan la dificultad de alcanzarla: "La actitud crítica tiene que ser hacia uno mismo, no hacia los demás, y debe existir un compromiso ante el trabajo".

Destacan dos argumentos para rechazar la necesidad de tener actitud crítica: “„NO! Objetivar es imposible. Sobra este adjetivo", y el segundo argumento indica: "Debería ser una regla o acuerdo generalizado, pero que no todos lo siguen".

La interrogante que plantea la pregunta 5 es: " $i$ Cree que otra de las reglas sea tener aptitud metodológica?" Es decir, posea la capacidad para recurrir a procedimientos, instrumentos y técnicas pertinentes para probar sus supuestos.

Las respuestas afirmativas que dan los científicos sociales encuestados a la pregunta 5 es la más alta del estudio (93\%). Divididas las respuestas por disciplina, se encuentra que Administración y Contabilidad están totalmente de acuerdo con esta regla, en tanto que Antro- 
pología, Historia y Psicología muestran una aceptación muy alta $(89,90$ y $90 \%$ respectivamente) (figura 4$)$.

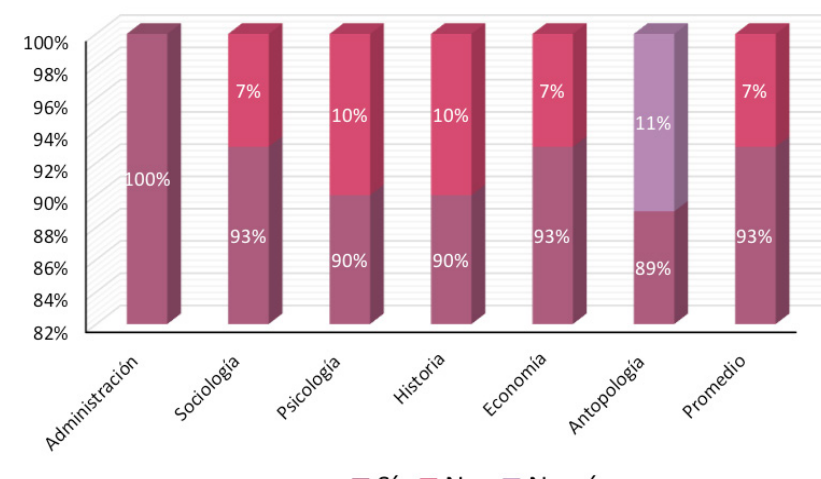

ní $\quad$ No No sé

Figura 4.

Respuesta a la pregunta 5. ¿Cree que otra de las reglas sea tener aptitud metodológica? Es decir, iposea la capacidad para recurrir a procedimientos, instrumentos y técnicas pertinentes para probar sus supuestos?

Debido a que existe un acuerdo general en todos los entrevistados que abarca a todas las disciplinas, se omitió hacer la prueba ji cuadrada; prácticamente no hay diferencias entre los científicos entrevistados.

Las opiniones que ratifican la importancia de este concepto son: "Los fenómenos son tan complejos que requieren de ciertas cualidades y aptitudes de los investigadores", "Definitivamente, para que la investigación se realice de manera ordenada", "Eso es el método científico", "Congruencia entre la teoría y el método y bajarla a la técnica, la instrumentación" o "No se trata de si el investigador quiera o no tenga aptitud. Tiene que ser así para evitar pseudo-investigaciones".

Aunque fueron pocos los que rechazaron esta regla, no dejaron de emitir su opinión: "No me gusta la palabra aptitud, más bien tendría que ser aprender las estrategias metodológicas, para irse apropiando de estas reglas y acuerdos"; otro condicionó la regla: "No se cumple cuando los resultados de la investigación afectan a la iniciativa privada o al gobierno".

La pregunta 6 del cuestionario es: " $i$ Considera que otra de las reglas sería que el científico esté dispuesto a comunicar los resultados encontrados de manera abierta?" Es decir, de forma verificable o replicable.

Los investigadores encuestados que están de acuerdo en que "Publicar de manera abierta" es una regla de la investigación científica alcanzan $88 \%$, en promedio; el resto la rechazan. Los psicólogos encuestados son los que más la rechazan (20\%), y los que menos la rechazan son los economistas, administradores y contadores (7 y $9 \%$ respectivamente) (figura 5 ).

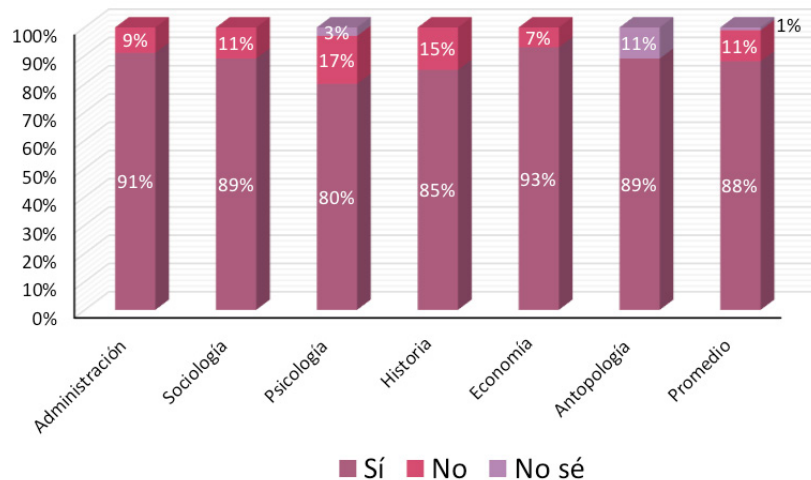

Figura 5.

Respuestas a la pregunta 6. ¿Considera que otra de las reglas sería que el científico esté dispuesto a comunicar los resultados encontrados de manera abierta? Es decir, ¿verificable o replicable?

Algunos de los encuestados explicaron su importancia: "Claro, tratar de verificar estos resultados es el propósito", "Para discusión y enriquecimiento del mismo resultado", "El logro del investigador es obtener resultados y exponerlos", "También de manera pública y gratuita", "Es un compromiso de todo investigador con la comunidad científica y la sociedad" o "Para que sea reconocido y evaluado".

Las opiniones que niegan la validez de este acuerdo expresaron: "No me gusta la palabra verificable; mostrar más que demostrar; discernir más que verificar", "En ciencias sociales es imposible la replicación" o "Si publicar, en el sentido de difusión, comunicación; no en términos de verificación o replicación".

\section{Discusión}

Las opiniones de los científicos manifestadas en esta investigación sólo representan disposiciones para la acción, es decir, no son necesariamente las acciones que ellos siguen cuando hacen sus investigaciones. Medawar (2013) lo advierte: "No es fácil, ni siempre será necesario, trazar una distinción clara entre los científicos que 'realmente investigan' y los que efectúan operaciones científicas, al parecer de memoria" (pp. 46). No obstante, el que los científicos en activo den su opinión acerca de las reglas que guían su desempeño tiene la ventaja de ser una fuente directa respecto a la investigación científica, el objeto de estudio de esta indagación.

La finalidad de esta investigación fue parcialmente resuelta; ahora se tiene una idea cuantitativa de la importancia que tiene la investigación científica para los investigadores de las disciplinas sociales; sin embargo, es necesario reconocer la existencia de un número 
significativo de investigadores que todavía rechaza la idea de hacer investigaciones científicas en sus respectivas disciplinas, con las implicaciones que tiene para la formación de los alumnos. Por otra parte, las pruebas aportadas para algunas disciplinas (antropólogos e historiadores) necesitan ampliarse para que cumplan los requisitos de la prueba ji cuadrada, por lo que se recomienda ampliar estas muestras.

Respecto a los dos primeros supuestos de esta investigación, cabe afirmar que se corroboró la idea de Medawar y Schrödinger en el sentido de que los investigadores no expresan de manera espontánea las reglas de la investigación; sin embargo, la mayoría de ellos coincide en aceptar que las preguntas directas que se les hicieron se refieren a las reglas de la investigación científica. La tercera suposición fue parcialmente verificada porque entre los investigadores de tiempo completo de las diferentes disciplinas de ciencias sociales no se percibe que exista entre ellos una diferencia estadísticamente significativa, excepto en Sociología, Psicología y Economía, y sólo en algunas reglas. En todo caso, es recomendable ampliar la muestra de cada disciplina para poder discernir con mayor confiabilidad sus diferencias, si es que existen.

Otro elemento que vale la pena mencionar es que la investigación utilizó nuevos conceptos. El más original es "Aptitud metodológica", registrado en la pregunta 4 del cuestionario. Esta categoría concentra todas las destrezas y habilidades implicadas en la prueba de las hipótesis (conocimientos, procedimientos, instrumentos y técnicas). Su desarrollo se debió a que varios científicos habían testimoniado que no era posible concentrar todas las pericias y experiencias correspondientes a esta parte de su trabajo profesional; como Budker (en Keldysh, 1982), físico experimental, escribe: "[Existe un] conjunto de elementos de cada día y cada hora de trabajo que no están descritos en manuales, ni en monografías, ni pueden ser siquiera descritos en ellos" (pp. 129).

Otra categoría novedosa a la que se recurrió asoció la disposición para hacer algo (la actitud) con el proceder que debiera asumir el científico frente a los hechos que investiga (ser objetivo, racional, buscar la verificabilidad, etcétera). De ambas categorías se desarrolló el concepto "actitud crítica". Ambos conceptos (aptitud metodológica y actitud crítica) fueron aceptados por la gran mayoría de los científicos encuestados como elementos que describen las reglas de su trabajo profesional. El reconocimiento por parte de los científicos de ambos conceptos, en esencia nuevos, refutaría la peregrina idea de que las respuestas de los científicos obedecen a una tradición o visión compartida de lo que consideran que es su activi- dad, por la sencilla razón de que no tienen correspondencia con la concepción tradicional de la ciencia.

Es necesario hacer una observación adicional: los resultados encontrados en esta investigación son potencialmente valiosos para los estudiantes de educación superior en ciencias sociales. Si las cuatro reglas aceptadas mayoritariamente por los científicos se hicieran explícitas, quizá se les haría menos compleja la práctica de la investigación científica.

\section{ConcLusiones}

Este estudio piloto demuestra, provisionalmente, que de manera espontánea o libre los científicos sociales no mencionan alguna regla de la investigación científica que produzca un acuerdo mayoritario entre los científicos sociales; excepto entre los sociólogos, quienes en su mayoría aceptan que una regla es "seguir el método científico".

Respecto al examen de las reglas recordadas con preguntas directas o ayudadas es posible afirmar, de manera provisional, que son aceptadas y reconocidas como reglas de la investigación científica por la gran mayoría de los científicos sociales sin importar la disciplina de que se trate. Sin embargo, existe un sector minoritario, pero significativo estadísticamente, de los científicos sociales que rechaza dos de las cuatro reglas de la investigación científica. Las explicaciones más reiterativas de los investigadores se relacionan con las teorías de los estudios culturales y la teoría crítica (Harré y Krausz, 1996; Adorno, 2001).

\section{Conclusiones por disciplinas}

En Psicología (incluye Psicología Social), los investigadores muestran las mayores discrepancias frente a los promedios de las otras disciplinas en dos reglas de la investigación científica; una manifestada de manera espontánea: "Tener ética o bioética"; una minoría de psicólogos la mencionan, en tanto que para el resto de las disciplinas no existe como regla o compromiso. La segunda diferencia se manifestó en las preguntas ayudadas. Entre los psicólogos existe un sector estadísticamente significativo que rechaza que su disciplina estudie la realidad tal cual.

Los sociólogos y politólogos presentan diferencias significativas frente a las otras disciplinas en tres reglas mencionadas de manera espontánea; 1) son los exponentes mayoritarios de la idea de que la ciencia requiere del método científico; 2) una fuerte minoría recuerda la necesidad de contar con instrumentos y técnicas especializadas, y 3) hay pocos sociólogos que aceptan que "no existen reglas" en la investigación científica. Cuan- 
do se mencionan de modo explícito las reglas expuestas en las preguntas ayudadas, sus respuestas se apegan a los promedios encontrados en tres de las cuatro reglas; excepto en tener actitud crítica, son los científicos sociales que más la rechazan.

En el caso de Antropología, sus investigadores son los que más discrepan del resto de los científicos sociales cuando expresan de manera espontánea sus opiniones acerca de las reglas de la investigación científica: "seguir el método científico" y "tener actitud crítica" están por debajo de la aceptación del resto de los científicos de las otras disciplinas; por el contrario, "seguir leyes y reglamentos institucionales" y "tener que publicar" están por encima del resto. En cuanto a los antropólogos, se recomienda ampliar la muestra porque es la que tiene el menor número de profesionales encuestados.

Los investigadores de Administración de Empresas y Contabilidad tienen un comportamiento contradictorio respecto a las reglas manifestadas de modo espontáneo; existen minorías significativas opuestas en aspectos significativos de la investigación científica. Una proporción considerable de ellos opina que existen reglas dentro de ella, y otros, en una proporción casi semejante, no creen en su existencia.

En sus recuerdos espontáneos de las reglas de la investigación científica, los economistas se mantienen dentro del promedio general. Respecto a los recuerdos ayudados, en tres de las cuatro reglas puestas a su consideración sus opiniones se mantienen en los promedios generales. En la regla "Estudiar la realidad" sus opiniones favorables a esta regla son significativamente mayores al resto de los colegas de las otras disciplinas estudiadas.

La reflexión que conllevan estos resultados está asociada con la formación de los futuros profesionales de la ciencia social. Cuando hay tantas discrepancias, uno se pregunta si los estudiantes reciben la capacitación adecuada para hacer frente a los retos de la compleja realidad. Esta es una época en que es necesario dejar de buscar soluciones intuitivas o empíricas y acercarse a soluciones más generales, que sólo es capaz de proporcionar la investigación científica.

\section{Referencias}

Adorno, T. (2001), Epistemología y ciencias sociales. España: Universidad de Valencia/Cátedra.

Bourdieu, P. (2000), Los usos sociales de la ciencia. Buenos Aires: Nueva Visión.

Danaher, P. J., \& Mullarkey, G. W. (2003). Factors affecting online advertising recall: A study of students. Journal of Advertising Research, 43 (03), 252-267.

David, B. J. (2006), The Scientist's Role in the Society: a
Comparative Study. Nueva Jersey: Prentice-Hall.

Edward E. J y Harold B. G. (1980). Fundamentos de Psicología Social. México, Limusa.

El Muestreo-Estadística. (2017) http://www.estadistica.mat. uson.mx/Material/elmuestreo.pdf pp. 1-5. acceso: 02/01/2017.

Fiske, S. T., Gilbert, D. T., \& Lindzey, G. (eds.). (2010). Handbook of Social Psychology. Vol. 2. John Wiley \& Sons.

Freeland J. H. (2006) Anatomía del fraude científico, Barcelona, Crítica.

Gould, S. J. (2004) Érase una vez el zorro y el erizo, las humanidades y la ciencia en el tercer milenio, Barcelona, Crítica.

Harré, R. \& Krausz, M. (1996). Varieties of relativism. Oxford: Blackwell.

Hempel, C. G. (1988). La explicación científica. Estudios sobre la filosofía de la ciencia. México: Paidós.

Holton, G. (1985). La imaginación científica. México: Fondo de Cultura Económica.

Keldysh, M. V. \& Artobolevsky, I. (1982.). La edad del conocimiento. México: Guajardo.

Knorr-Cetina, K. (1999), Epistemic cultures. How sciences make knowledge. England, London, Cambridge, Harvard University Press.

Lama García, A. (2011). ¿Existen reglas implícitas dentro de la investigación científica?. Revista de la educación superior. Vol. XL (4), núm. 160, octubre-diciembre, pp. 73-93.

Lama García, A., Del Castillo Mussot, M., \& Lama Zubirán, M. A. (2013). ¿Existen diferencias en las creencias que regulan las investigaciones científicas de los científicos naturales y sociales? 185 investigadores responden. En Argumentos. Estudios críticos de la sociedad, UAM, Xochimilco, núm. 71, enero-abril, pp. 39-66.

Latour, B. (1987). Science in action. How to follow scientist and engineers trough society. Cambridge, Massachusetts: Harvard University Press.

Medawar, P. (2013). Consejos a un joven científico. México: Fondo de Cultura Económica.

Merton, R. (1973). The sociology of science: Theoretical and empirical investigations. Chicago: The University Press.

Merton, R. (2016). Manifest and latent functions social. En Wesley Longhofer and Daniel Winchester (eds.). Theory re-wire: New connections to classical and contemporary perspectives. New York and London: Routledge, pp. 68-84.

Reinares-Lara, E., Reinares-Lara, P., \& Olarte-Pascual, C. (2016). Formatos de publicidad no convencional en televisión versus spots: Un análisis basado en el recuerdo. En Historia y Comunicación Social, 21 (1), 257. Disponible en https://doi.org/10.5209/rev_HICS.2016.v21.n1.52695

Pérez Tamayo, R. (2008). La estructura de la ciencia. México: Fondo de Cultura Económica.

Schrödinger, E. (1997). La naturaleza y los griegos. Madrid: Tusquets. 
Siegel, S. \& Castellan, N. J. (1998). Estadística no paramétrica. Aplicada a las ciencias sociales, 4a. ed. México: Trillas.

Thuillier, P. (1991). De Arquímides a Einstein. Las caras ocultas de la invención científica. México: Conaculta/Alianza Editorial.

Thurow, L. C. (1996). El futuro del capitalismo. Argentina: Javier Vergara.

Weiers, R. M. (1986). Investigación de mercados. México:
Prentice-Hall-Hispanoamericana.

Wigglesworth, V. B. (1987). El control de la forma en el cuerpo.

En Ronald Duncan y Miranda Weston-Smith (comps.). La enciclopedia de la ignorancia. México: Fondo de Cultura Económica.

Villoro Toranzo, L. (2014). La universidad, un aparato ideológico. En Casa del tiempo, año XXXIII, vol. 1, núm. 3, abril. 


\section{APÉNDICE}

\section{Cuestionario utilizado}

Buenos días, tardes etc.

Somos un grupo interdisciplinario de estudiantes y especialistas de diversas universidades que estamos interesados en conocer las opiniones de los expertos en torno a su trabajo profesional. ¿Sería tan amable de contestar seis breves preguntas? Muchas gracias.

¿Es Ud. investigador o profesor investigador de Tiempo Completo? Si ( ) No ( ) cancelar entrevista En qué carrera o instituto labora

De qué Universidad (y país si no es México)

1. En su opinión ¿Cree que existan reglas o acuerdos dentro de la investigación científica que sigan la mayoría de los científicos en activo?

$$
\text { Si ( ) No ( ) pasar a la p. } 3 \quad \text { No sé ( ) pasar a la p. } 3
$$

2. ¿Podría mencionar algunas de estas reglas o acuerdos? $\quad$ No las recuerdo ( )

3. ¿Cree que una de las reglas sea pensar que en la naturaleza (o en la sociedad, en su caso) existen leyes o regularidades que pueden ser explicadas a través de la observación y el razonamiento?

$$
\mathrm{Si}(\mathrm{No}(\mathrm{N} \quad \text { No sé ( ) }
$$

4. ¿Estima que otra de las reglas sería que el investigador tenga actitud crítica frente al objeto de estudio? Es decir, que desarrolle la capacidad de analizar de manera objetiva, racional, verificable y sistemática la información contenida en toda investigación.

$$
\text { Si ( ) No ( ) No sé ( ) }
$$

5. ¿Cree que otra de las reglas sea tener aptitud metodológica? Es decir, posea la capacidad para recurrir a procedimientos, instrumentos y técnicas pertinentes para probar sus supuestos.

$$
\mathrm{Si}(\mathrm{No}(\mathrm{l}) \quad \text { No sé }(\mathrm{)}
$$

6. ¿Considera que otra de las reglas sería que el científico esté dispuesto a comunicar los resultados encontrados de manera abierta? Es decir, verificable o replicable.

$$
\text { Si ( ) No ( ) No sé ( ) }
$$

Por último ¿Cuál es el grado máximo de sus estudios estudió su posgrado? En qué país en qué especialidad ¿En qué institución

Con el objeto de informarle del resultado de esta investigación y eventualmente para fines de supervisión de este cuestionario ¿Podría proporcionarnos su mail?

Esto es todo. Muchas gracias.

El entrevistado es

El entrevistado es menor de 40 años ( ) más de 40 años ( )

hombre ( ) mujer ( ) 


\section{Meta-Análisis del Artículo}




\section{Dimensión Cuantitativa}

\section{Perfil de Evaluación entre pares}
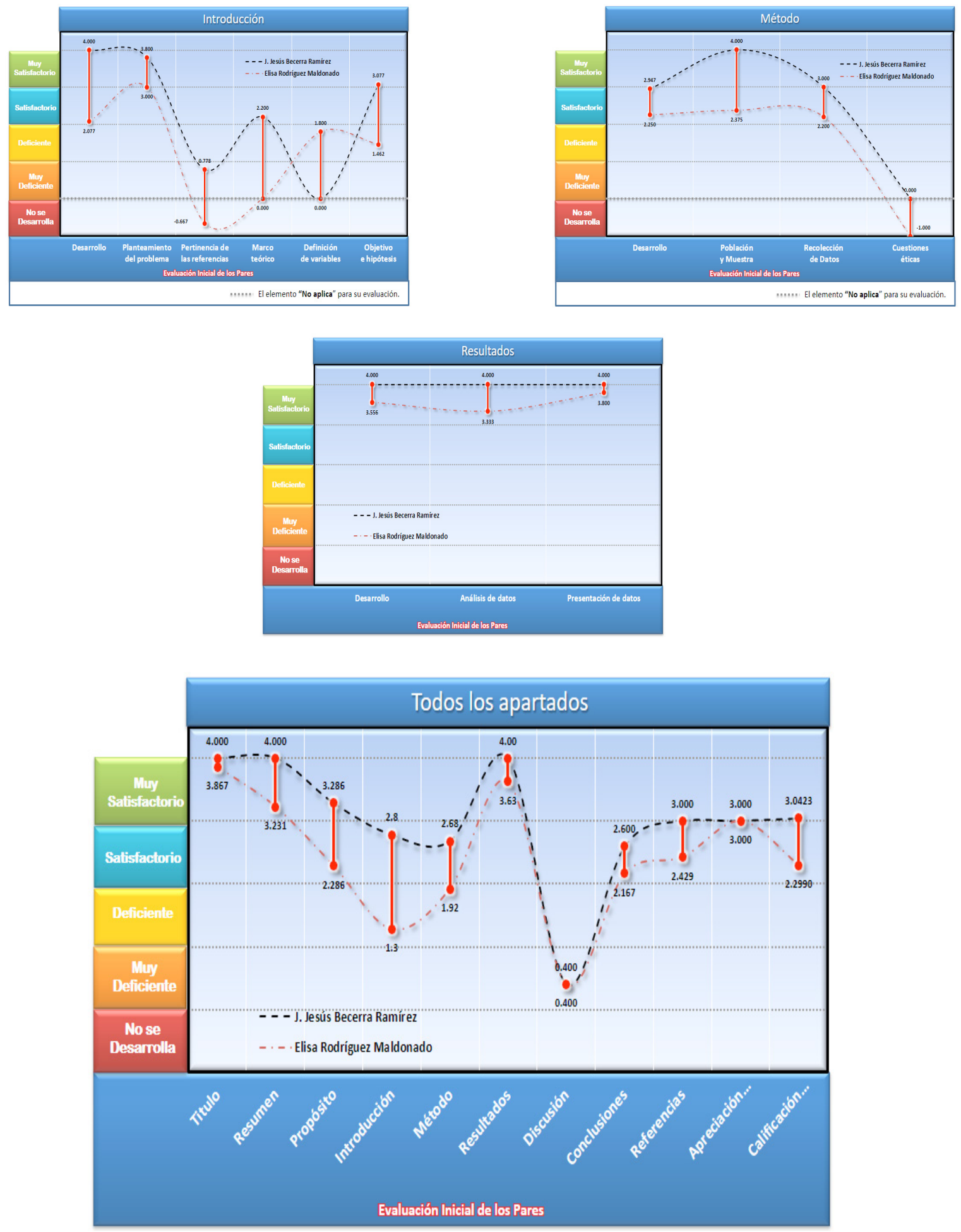


\section{Índice de Concordancia}

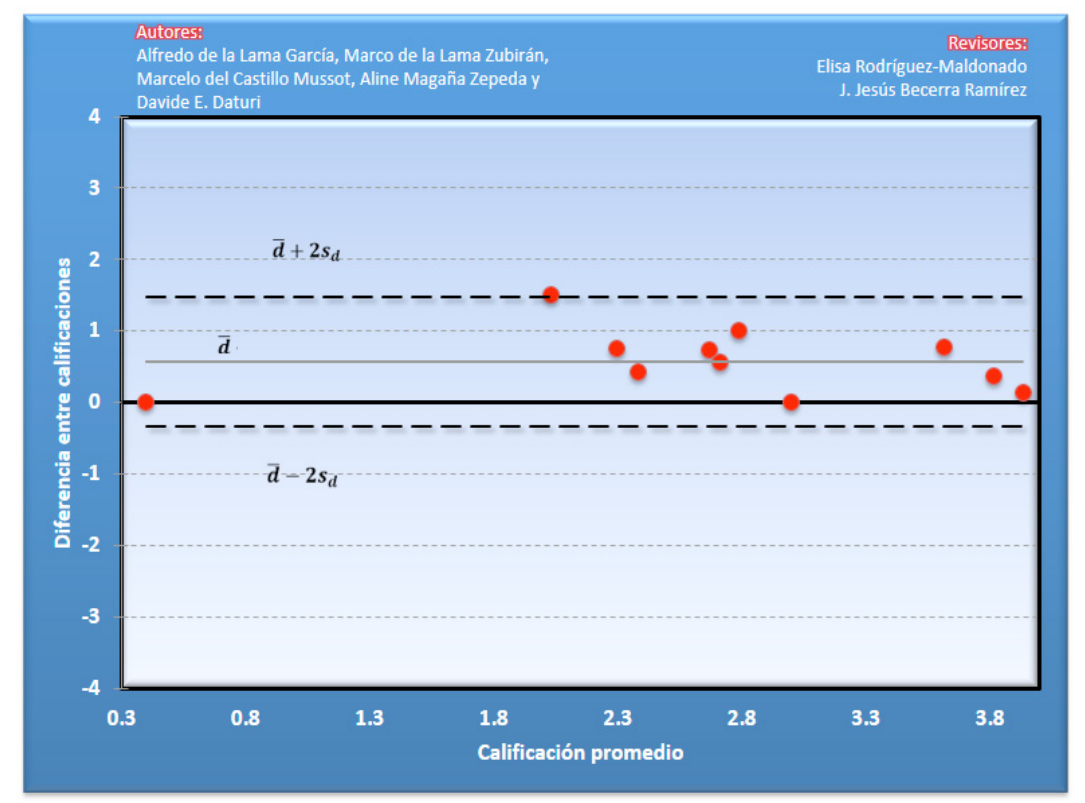

Índice de Acuerdo

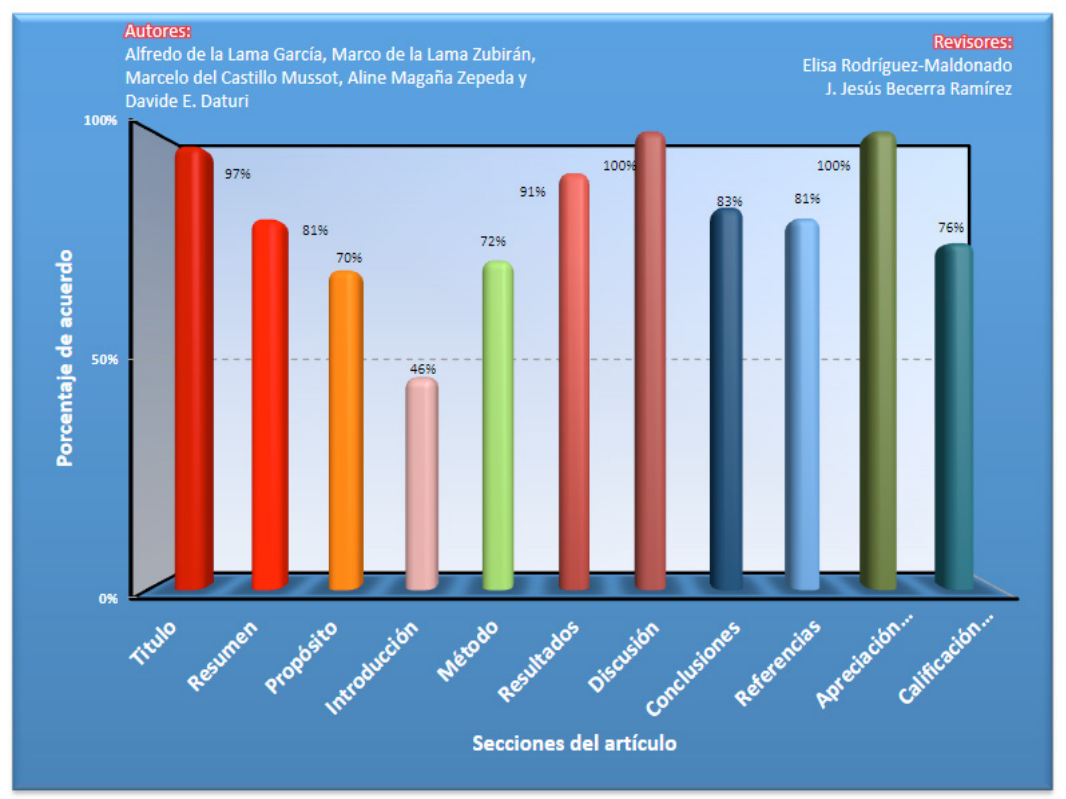


Dimensión Cualitativa

\begin{tabular}{|c|c|}
\hline Revisor 1 & Revisor 2 \\
\hline Elisa Rodríguez-Maldonado & J. Jesús Becerra Ramírez \\
\hline \multicolumn{2}{|c|}{ Título/Autoría } \\
\hline $\begin{array}{l}\text { Cambiar el orden de las palabras en la pregunta. Se su- } \\
\text { giere que diga: ¿Son similares las opiniones sobre las } \\
\text { reglas de investigación? }\end{array}$ & $\begin{array}{l}\text { El título es concreto y cumple con las características } \\
\text { solicitadas, así como la justificación es adecuada para } \\
\text { abordar la temática. }\end{array}$ \\
\hline \multicolumn{2}{|c|}{ Resumen } \\
\hline Ver comentarios en el documento revisado. & $\begin{array}{l}\text { El apartado cumple con los criterios solicitados, es claro } \\
\text { y accesible de entender. }\end{array}$ \\
\hline \multicolumn{2}{|c|}{ Próposito del Estudio } \\
\hline Ver comentarios en el documento revisado. & $\begin{array}{l}\text { En el proyecto queda claro el propósito y la importancia } \\
\text { de trabajar el tema. }\end{array}$ \\
\hline \multicolumn{2}{|c|}{ Introducción } \\
\hline $\begin{array}{l}\text { Es necesario ampliar marco de referencia, trasfondo y } \\
\text { revisión de la literatura científica sobre el tema estudia- } \\
\text { do en esta introducción. Diálogo con otros teóricos, y } \\
\text { hallazgos de otros investigadores, conclusiones, interro- } \\
\text { gantes, controversias, breve historial de estudios previos } \\
\text { relacionados a los planteamientos de esta investigación. } \\
\text { Mencionar qué sucede con las variables relacionadas en } \\
\text { contextos similares a los de la muestra seleccionada. Ver } \\
\text { comentarios en el documento revisado. }\end{array}$ & $\begin{array}{l}\text { Se recomienda buscar mayor información sobre hallazgos } \\
\text { de otros proyectos similares. }\end{array}$ \\
\hline \multicolumn{2}{|c|}{ Método } \\
\hline $\begin{array}{l}\text { Ampliar sobre el tipo de análisis utilizados para las pregun- } \\
\text { tas abiertas. Añadir información de validez del cuestionario. } \\
\text { Ver sugerencias y comentarios en el documento revisado. }\end{array}$ & $\begin{array}{l}\text { Se sugiere trabajar con consentimiento informado y desa- } \\
\text { rrollas la parte ética del proyecto, pues no se ve reflejada. }\end{array}$ \\
\hline
\end{tabular}


Artículo Empírico | Entre científicos de diferentes disciplinas sociales... de la Lama-García, de la Lama-Zubirán, et al.

\begin{tabular}{|c|c|}
\hline Revisor 1 & Revisor 2 \\
\hline \multicolumn{2}{|c|}{ Resultados } \\
\hline $\begin{array}{l}\text { Para algunas variables no hubo suficiente información para } \\
\text { por concluir. Ver comentarios en el documento revisado. }\end{array}$ & $\begin{array}{l}\text { En general me parece que realizan un trabajo adecuado y } \\
\text { de calidad. }\end{array}$ \\
\hline \multicolumn{2}{|c|}{ Discusión } \\
\hline $\begin{array}{l}\text { Habrá que ampliar la discusión. Cada hallazgo debe ser } \\
\text { discutido. ¿Se cumplieron los objetivos? ¿Se sostienen } \\
\text { las hipótesis formuladas? ¿Cómo ubican los resultados } \\
\text { del estudio en cuanto a lo ya investigado por otros au- } \\
\text { tores en temas similares o, en su defecto, en materias } \\
\text { relacionadas? Comparar los hallazgos propios con los } \\
\text { de otros autores, citar las referencias de otros estudios } \\
\text { que se utilicen en la comparación. Discutir errores me- } \\
\text { todológicos con sus posibles soluciones. Exponer cuá- } \\
\text { les resultados eran esperados y no esperados. ¿Cuáles } \\
\text { son los alcances de la aplicación de los resultados? Ver } \\
\text { comentarios en el documento revisado. }\end{array}$ & $\begin{array}{l}\text { Se recomienda ampliar la discusión pues los hallazgos se } \\
\text { presentan de forma general. }\end{array}$ \\
\hline \multicolumn{2}{|c|}{ Conclusiones } \\
\hline Ver comentarios en el documento revisado. & $\begin{array}{l}\text { En general se encuentra bien desarrollado, pero se reco- } \\
\text { mienda retomar mayores antescedentes. }\end{array}$ \\
\hline \multicolumn{2}{|c|}{ Referencias } \\
\hline Ver comentarios en el documento revisado. & $\begin{array}{l}\text { En general se encuentra bien desarrollado, pero se reco- } \\
\text { mienda retomar mayores antescedentes }\end{array}$ \\
\hline
\end{tabular}




\section{Historia del Proceso}

\section{EDITORIAL}

